\title{
Combined total variation of first and fractional orders for Poisson noise removal in digital images
}

\author{
Pham Cong Thanga , PhD, Lecturer, orcid.org/0000-0002-6428-102X, pcthang@dut.udn.vn \\ Tran Thi Thu Thaob, MSc., Lecturer, orcid.org/0000-0001-7705-2405 \\ Pham Minh Tuana, PhD, Lecturer, orcid.org/0000-0001-9843-9676 \\ Nguyen Thanh Conga, MSc., Specialist, orcid.org/0000-0002-8060-0238 \\ aThe University of Danang - University of Science and Technology, 54, Nguyen Luong Bang St., 550000, \\ Danang, Vietnam \\ bThe University of Danang - University of Economics, 71, Ngu Hanh Son St., 550000, Danang, Vietnam
}

\begin{abstract}
Introduction: Many methods have been proposed to handle the image restoration problem with Poisson noise. A popular approach to Poissonian image reconstruction is the one based on total variation. This method can provide significantly sharp edges and visually fine images, but it results in piecewise-constant regions in the resulting images. Purpose: Developing an adaptive total variation-based model for the reconstruction of images contaminated by Poisson noise, and an algorithm for solving the optimization problem. Results: We proposed an effective way to restore images degraded by Poisson noise. Using the Bayesian framework, we proposed an adaptive model based on a combination of first-order total variation and fractional order total variation. The first-order total variation model is efficient for suppressing the noise and preserving the keen edges simultaneously. However, the first-order total variation method usually causes artifact problems in the obtained results. To avoid this drawback, we can use high-order total variation models, one of which is the fractional-order total variation-based model for image restoration. In the fractional-order total variation model, the derivatives have an order greater than or equal to one. It leads to the convenience of computation with a compact discrete form. However, methods based on the fractional-order total variation may cause image blurring. Thus, the proposed model incorporates the advantages of two total variation regularization models, having a significant effect on the edge-preserving image restoration. In order to solve the considered optimization problem, the Split Bregman method is used. Experimental results are provided, demonstrating the effectiveness of the proposed method. Practical relevance: The proposed method allows you to restore Poissonian images preserving their edges. The presented numerical simulation demonstrates the competitive performance of the model proposed for image reconstruction. Discussion: From the experimental results, we can see that the proposed algorithm is effective in suppressing noise and preserving the image edges. However, the weighted parameters in the proposed model were not automatically selected at each iteration of the proposed algorithm. This requires additional research.
\end{abstract}

Keywords - total variation, image restoration, Poisson noise, minimization method.

For citation: Pham C. T., Tran T. T. T., Pham M. T., Nguyen T. C. Combined total variation of first and fractional orders for Poisson noise removal in digital images. Informatsionno-upravliaiushchie sistemy [Information and Control Systems], 2021, no. 5, pp. 10-19. doi:10.31799/1684-8853-2021-5-10-19

\section{Introduction}

Poisson noise is known as photon noise or shot noise [1]. Poisson noise removal is an important task in various applications such as electronic microscopy [2, 3], tomography [4, 5], X-ray [6, 7], etc. In electronic microscopy imaging, the number of electrons collected to create an image pixel follows the Poisson distribution [8]. It means that parts of an image with higher intensity have a higher noise level than parts with low intensity. A low signal-tonoise ratio leads to a high level of noise, which affects detected edges and accuracy in measurement $[9,10]$. With the electron tomography technique, data acquisition is the detection of scattered electron particles. The emission and subsequent detection of electrons follow a Poisson process [11]. These processes degrade data quality due to Poisson noise $[12,13]$. Meanwhile, X-ray is a popular medical imaging technique. X-rays are produced using photons, which have very small wavelengths and high penetration ability [14]. X-ray images are produced using a digital receptor. The photon scattering follows Poisson noise distribution which leads to degradation of the $\mathrm{X}$-ray image quality and to the difficulty of diagnosis [15].

For many years, many variational methods have been proposed to handle the restoration problem with Poisson noise [16-23]. One of variational models for Poissonian image reconstruction is the one based on the total variation (TV) norm as regularization term [24]:

$$
\min _{z \in \Omega}\left(\|z\|_{T V}+\beta\langle 1, K z-f \log K z\rangle\right)
$$

where $\beta$ is a positive regularization parameter; $z$ must be positive in $\Omega ; K$ is a blurring operator, the operator $\|z\|_{T V}$ stands for the TV of $u$. Authors in [25] replaced the TV regularization term in (1) by $\|z\|_{T V}+\frac{\lambda}{2}\|z\|_{2}^{2}$ and proposed bounded varia- 
tion-based Poissonian images restoration model as follows (TV-model):

$$
z^{*}=\underset{z \in \Omega}{\arg \min }\left(\|z\|_{T V}+\frac{\lambda}{2}\|z\|_{2}^{2}+\beta\langle 1, K z-f \log K z\rangle\right) .
$$

The model (2) performs very well for preserving edges while removing noise. However, it often causes undesired artifact effects in smooth regions. To overcome these effects, some high-order models have been introduced for restoring blurred images corrupted by Poisson noise. The authors in [26] replaced the term $\|z\|_{T V}$ in (2) with higher-order $\|z\|_{H T V}$ and proposed following model (HTV-model):

$$
z^{*}=\underset{z \in \Omega}{\arg \min }\left(\|z\|_{H T V}+\frac{\lambda}{2}\|z\|_{2}^{2}+\beta\langle 1, z-f \log z\rangle\right) .
$$

Recently, fractional-order derivatives are widely applied in image processing [27-31]. The works have reflected the good performance of the fractional-order derivative in image denoising with edge-preserving. Following [29], the fractional-order TV model for Poissonian image denoising and deblurring is as follows (FTVmodel):

$$
z^{*}=\underset{u}{\arg \min }\left(\|z\|_{T V_{\alpha}}+\beta\langle 1, K z-f \log K z\rangle\right),
$$

where $\beta$ is positive parameter; $\|z\|_{T V_{\alpha}}$ stands for fractional-order TV defined in Eq. (10).

Motivated by the above studies, we introduce an adaptive TV based optimization problem as follows:

$$
\begin{gathered}
z^{*}=\underset{z \in S(\Omega)}{\arg \min } E(z), \\
z^{*}=\underset{z \in \Omega}{\arg \min }\left(\mu_{1}\|z\|_{T V}+\mu_{2}\|z\|_{T V_{\alpha}}+\right. \\
\left.+\frac{\lambda}{2}\|z\|_{2}^{2}+\beta\langle 1, K z-f \log K z\rangle\right),
\end{gathered}
$$

where $\mu_{1}, \mu_{2}$ and $\beta$ are positive paramters, $\mu_{1}$, $\mu_{2} \in(0,1)$.

Motivated by the previous works, we propose a Poisson noise removal model that can substantially reduce artifact effects while preserving edges in the restored images. The proposed model is designed by combining advantages of the first order TV and fractional order TV. We extend the split Bregman method for solving the optimization problem. Furthermore, we provide experimental results to demonstrate the efficiency of our algorithm for the considered problem, in comparison with state-of-the-art methods.

\section{Preliminaries}

We recall the principle behind Eq. (5). We aim at reconstructing the original image $u$ with the known noisy image $f$. Our strategy is to find the image $u$ which maximizes the conditional probability $P(u \mid f)$. Bayes's rule gives

$$
P(z \mid f)=\frac{P(f \mid z) P(z)}{P(f)} .
$$

The probability density function of the observed image $f$ corrupted by Poisson noise is:

$$
P(f \mid z)=\frac{z^{f} \exp (-z)}{f !} .
$$

Suppose that $f$ has size $M \times N$, and let $\mathcal{I}=\{1, \ldots$, $M\} \times\{1, \ldots, N\}$ denote the domain of $f$. For $i \in \mathcal{I}$, we write $f_{i}$ the pixel of $f$ at position $i$ (and similarly $u_{i}$ the pixel of $u$ at position $i$ ) [32]. Then:

$$
P(f \mid z)=\prod_{i \in I} \frac{\left(z_{i}\right)^{f_{i}} e^{\left(-z_{i}\right)}}{f_{i} !} .
$$

Maximizing $P(z \mid f)$ is equivalent to minimizing $-\log (P(z \mid f))$, so let us compute the quantity $-\log (P(f \mid z))$ :

$$
\sum_{i \in I} z_{i}-f_{i} \log \left(z_{i}\right)+\log \left(f_{i} !\right)
$$

Since $f$ is constant, we can ignore the term $\log \left(f_{i} !\right)$. Now we assume that $P(z)$ follows a choice of the prior:

$$
P(z)=\exp \left(-\frac{1}{\tau}(\phi(z))\right.
$$

where $\tau$ is a normalization factor being positive and constant.

In this work, we assumpt that

$$
\phi(z)=\mu_{1}\|z\|_{T V}+\mu_{2}\|z\|_{T V_{\alpha}}+\frac{\alpha}{2}\|z\|_{2}^{2} .
$$

The assumption on $P(u)$ means that each pixel depends (weakly) on the neighbouring pixels only, so we do not lose much by assuming independence.

We now have all the ingredients to maximize $P(z \mid f)$. By Eq. (6), this amounts to minimize the expression $-\log (P(f \mid z))-\log (P(z))$, so we can plug in Equations (7) and (8) to get:

$$
z^{*}=\underset{z}{\arg \min } \sum_{i \in I}\left(\frac{1}{\tau} \phi\left(z_{i}\right)+\left(z_{i}-f_{i} \log \left(z_{i}\right)\right)\right)
$$


and we can view this expression as a discrete approximation of the functional $E(\cdot)$ defined as

$E(z)=\left(\mu_{1}\|z\|_{T V}+\mu_{2}\|z\|_{T V_{\alpha}}+\frac{\alpha}{2}\|z\|_{2}^{2}+\beta\langle 1, z-f \log z\rangle\right)$,

where $\beta=\tau$ is positive and constant parameter.

In case of the blur effect, we can generalize the model (5) for restoring a blurred image corrupted by Poisson noise as follows:

$$
\begin{aligned}
& E(z)=\left(\mu_{1}\|z\|_{T V}+\mu_{2}\|z\|_{T V_{\alpha}}+\right. \\
& \left.+\frac{\alpha}{2}\|z\|_{2}^{2}+\beta\langle 1, K z-f \log K z\rangle\right)
\end{aligned}
$$

where $\beta$ is positive and constant parameter.

The discrete gradients of an image $u$ for the pixel location $(i, j)$ in $z(i=1 . . M ; j=1 . . N)$ are defined like [33-35]:

$$
\begin{gathered}
\|z\|_{T V}=\sqrt{\left(\nabla_{1} z\right)^{2}+\left(\nabla_{2} z\right)^{2}} \\
\nabla_{1} z_{i, j}=z_{i+1, j}-z_{i, j}, \nabla_{2} z_{i, j}=z_{i, j+1}-z_{i, j} \\
\nabla_{11} z_{i, j}=z_{i+1, j}-2 z_{i, j}+z_{i-1, j} \\
\nabla_{22} z_{i, j}=z_{i, j+1}-2 z_{i, j}+z_{i, j-1} \\
\nabla_{12} z_{i, j}=\nabla_{21} z_{i, j}=z_{i, j}-z_{i, j-1}-z_{i-1, j}+z_{i-1, j-1} \\
\|z\|_{H T V}=\sqrt{\left(\nabla_{11} z\right)^{2}+\left(\nabla_{12} z\right)^{2}+\left(\nabla_{21} z\right)^{2}+\left(\nabla_{22} z\right)^{2}}
\end{gathered}
$$

Due to the convenience in numerical implementation, from Greunwald - Letnikov (GL) fractional-order derivative, the discrete fractional-order TV $\|z\|_{T V_{\alpha}}$ of $z \in \Omega$ is defined as follows [27, 31]:

$$
\|z\|_{T V_{\alpha}}=\sqrt{\left(\nabla_{1}^{\alpha} z\right)^{2}+\left(\nabla_{2}^{\alpha} z\right)^{2}}
$$

where the discrete gradients $\nabla_{1}^{\alpha} z$ and $\nabla_{2}^{\alpha} z$ are defined as follows:

$$
\left(\nabla_{1}^{\alpha} z\right)_{i, j}=\sum_{k=0}^{L-1} C_{k}^{\alpha} z_{i-k, j},\left(\nabla_{2}^{\alpha} z\right)_{i, j}=\sum_{k=0}^{L-1} C_{k}^{\alpha} z_{i, j-k} .
$$

Parameter $L$ is the number of neighboring pixels that are used to compute the fractional-order derivative at each pixel; the coefficients $\left\{C_{k}^{\alpha}\right\}$ are defined as follows [27]:

$$
C_{k}^{\alpha}=\frac{\Gamma(\alpha+1)}{\Gamma(k+1) \Gamma(\alpha+1-k)}, k=1 . . L-1 .
$$

\section{Computational method}

In this section, we derive the numerical method for the problem (5) in detail. There are many meth- ods which can be employed to obtain the solution of the optimization problem. In this article, we decide to employ the split Bregman method for solving the optimization problem.

The split Bregman method performs break the minimization problem down into easy subproblems [36-38]. Subproblems can be directly solved with tools like fast Fourier transform (FFT), shrinkage operator that makes the optimization algorithm rather fast. We have a scalar $\gamma$ and two convex functionals $\Psi(\cdot)$ and $G(\cdot)$; and that we need to solve the following constrained optimization problem:

$$
\underset{z, w}{\arg \min }(\|w\|+\gamma G(z)), \text { s.t. } w=\Psi(z)
$$

We convert (11) into an unconstrained problem:

$$
\underset{z, w}{\arg \min }\left(\|w\|+\gamma G(z)+\frac{\rho}{2}\|d-\Psi(z)-b\|_{2}^{2}\right),
$$

where $w$ - splitting variable; $\rho$ is positive constant parameters; $b$ - variable of the Bregman iterations.

The solution to problem (12) can be approximated by the Split Bregman method:

$$
\begin{gathered}
z^{(k+1)}=\underset{z}{\arg \min }\left(\gamma G(z)+\frac{\rho}{2}\left\|w^{(k)}-\Psi(z)-b^{(k)}\right\|_{2}^{2}\right) \\
w^{(k+1)}=\underset{w}{\arg \min }\left(\|w\|_{1}+\frac{\rho}{2}\left\|w-\Psi\left(z^{(k+1)}\right)-b^{(k)}\right\|_{2}^{2}\right), \\
b^{(k+1)}=b^{(k)}+\Psi\left(z^{(k+1)}\right)-w^{(k+1)} .
\end{gathered}
$$

We return to the problem (5). By introducing three auxiliary variables $u, p$ and $q$, Eq. (5) is equivalent to the constrained optimization problem:

$$
\begin{gathered}
\min _{p, q, z, u}\left(\mu_{1}\|p\|+\mu_{2}\|q\|+\frac{\theta}{2}\|z\|_{2}^{2}+\beta\langle 1, u-f \log u\rangle\right), \\
\text { s.t. } p=\nabla z, q=\nabla^{\alpha} z, u=K z,
\end{gathered}
$$

where $\nabla z$ and $\nabla^{\alpha} z$ are defined in (9) and (10) respectively.

We convert the problem (5) to the unconstrained one as follows:

$$
\min _{p, q, z, u}\left(\begin{array}{c}
\mu_{1}\|p\|+\mu_{2}\|q\|+\frac{\theta}{2}\|z\|_{2}^{2}+ \\
+\beta\langle 1, u-f \log u\rangle+\frac{\rho_{1}}{2}\|p-\nabla z\|_{2}^{2}+ \\
+\frac{\rho_{2}}{2}\left\|q-\nabla^{\alpha} z\right\|_{2}^{2}+\frac{\rho_{3}}{2}\|u-K z\|_{2}^{2}
\end{array}\right),
$$

where $\mu_{1}, \mu_{2}, \beta$ and $\gamma$ are positive parameters; $\rho_{1}, \rho_{2}$ are Lagrangian multipliers. 
The extended split Bregman iterative method employed for solving the problem (5) can be described as follows:

$$
\left\{\begin{array}{c}
z^{(k+1)}=\underset{z}{\arg \min } \\
+\frac{\rho_{2}}{2}\left\|q^{(k)}-\nabla^{\alpha} z-b_{q}^{(k)}\right\|_{2}^{2}+ \\
+\frac{\rho_{3}}{2}\left\|u^{(k)}-K z-b_{z}^{(k)}\right\|_{2}^{2}
\end{array}\right)
$$

with update for $b_{p}^{(k+1)}, b_{q}^{(k+1)}, b_{z}^{(k+1)}$ :

$$
\left\{\begin{array}{l}
b_{p}^{(k+1)}=b_{p}^{(k)}+\nabla z^{(k+1)}-p^{(k+1)} \\
b_{q}^{(k+1)}=b_{q}^{(k)}+\nabla^{\alpha} z^{(k+1)}-q^{(k+1)} \\
b_{z}^{(k+1)}=b_{z}^{(k)}+K z^{(k+1)}-u^{(k+1)}
\end{array}\right.
$$

Hence, we have three subproblems to solve: $z, p$ and $q$.

Subproblem $z$. For the $z$ subproblem, optimality condition reads:

$$
\begin{gathered}
\theta z+\rho_{1}(\nabla)^{\mathrm{T}}\left(\nabla z+b_{p}^{(k)}-p^{(k)}\right)+ \\
+\rho_{2}\left(\nabla^{\alpha}\right)^{\mathrm{T}}\left(\nabla^{\alpha} z+b_{q}^{(k)}-q^{(k)}\right)=\mathbf{0} .
\end{gathered}
$$

Therefore, we have

$$
\begin{gathered}
\left(\theta+\rho_{1}(\nabla)^{\mathrm{T}} \nabla+\rho_{2}\left(\nabla^{\alpha}\right)^{\mathrm{T}} \nabla^{\alpha}\right) z= \\
=\rho_{1}(\nabla)^{\mathrm{T}}\left(p^{(k)}-b_{p}^{(k)}\right)+\rho_{2}\left(\nabla^{\alpha}\right)^{\mathrm{T}}\left(q^{(k)}-b_{q}^{(k)}\right) .
\end{gathered}
$$

The Eq. (15) can be solved efficiently with one Fourier transform operation and one inverse FFT operation as follows:

$$
z^{(k+1)}=F^{-1}\left(\begin{array}{c}
F\left(\rho_{1}(\nabla)^{\mathrm{T}}\left(p^{(k)}-b_{p}^{(k)}\right)+\right. \\
\frac{\left.+\rho_{2}\left(\nabla^{\alpha}\right)^{\mathrm{T}}\left(q^{(k)}-b_{q}^{(k)}\right)\right)}{\theta+\rho_{1} F\left((\nabla)^{\mathrm{T}} \nabla\right)+\rho_{2} F\left(\left(\nabla^{\alpha}\right)^{\mathrm{T}} \nabla^{\alpha}\right)}
\end{array}\right),
$$

where $F$ and $F^{-1}$ are the forward and inverse Fourier transform operators.
Subproblem $u$. For the u subproblem, optimality condition reads:

$$
\beta \frac{u-f}{u}+\rho_{3}\left(u-K z^{(k+1)}-b_{z}^{(k)}\right)=0
$$

At the $(k+1)$-th iteration, we compute $u$ by discretization scheme:

$$
\beta \frac{u^{(k+1)}-f}{u^{(k)}}+\rho_{3}\left(u^{(k+1)}-K z^{(k+1)}-b_{z}^{(k)}\right)=0 .
$$

Therefore, we have

$$
u^{(k+1)}=\frac{\beta f+\rho_{3} u^{(k)}\left(K z^{(k+1)}+b_{z}^{(k)}\right)}{\beta+\rho_{3} u^{(k)}} .
$$

Subproblems $p$ and $q$. The solution of the $p$ subproblem can readily be obtained by applying the soft thresholding operator:

$$
\begin{gathered}
p^{(k+1)}=\operatorname{shrink}\left(\nabla z^{(k+1)}+b_{p}^{(k)}, \frac{\mu_{1}}{\rho_{2}}\right)= \\
=\frac{\nabla z^{(k+1)}+b_{p}^{(k)}}{\left|\nabla z^{(k+1)}+b_{p}^{(k)}\right|} \cdot \max \left(\left|\nabla z^{(k+1)}+b_{p}^{(k)}\right|-\frac{\mu_{1}}{\rho_{1}}, 0\right) .
\end{gathered}
$$

The solution of the $q$ subproblem can also be obtained by applying the soft thresholding operator:

$$
\begin{gathered}
q^{(k+1)}=\operatorname{shrink}\left(\nabla^{\alpha} u^{(k+1)}+b_{p}^{(k)}, \frac{\mu_{2}}{\rho_{2}}\right)= \\
=\frac{\nabla^{\alpha} u^{(k+1)}+b_{p}^{(k)}}{\left|\nabla^{\alpha} u^{(k+1)}+b_{p}^{(k)}\right|} \cdot \max \left(\left|\nabla^{\alpha} u^{(k+1)}+b_{p}^{(k)}\right|-\frac{\mu_{2}}{\rho_{2}}, 0\right) .
\end{gathered}
$$

The complete method is summarized in Algorithm 1 .

Algorithm 1: Adaptive split Bregman method for solving the problem (5)

1. Initialize: $z^{(0)}=f ; p^{(0)}=q^{(0)}=0 ; \quad b_{p}^{(0)}=b_{q}^{(0)}=$ $=b_{z}^{(0)} ; k=1$

2. while $\left(\frac{\left\|u^{(k)}-u^{(k-1)}\right\|_{2}}{\left\|u^{(k)}\right\|_{2}}<\varepsilon\right) \|\left(k \leq N_{\max }\right)$ do

3. Calculate $z^{(k+1)}$ using (16)

4. Calculate $u^{(k+1)}$ using (17)

5. Calculate $p^{(k+1)}$ using (18)

6. Calculate $q^{(k+1)}$ using (19)

7. $b_{p}^{(k+1)}=b_{p}^{(k)}+\nabla z^{(k+1)}-p^{(k+1)}$

8. $b_{q}^{(k+1)}=b_{p}^{(k)}+\nabla^{\alpha} z^{(k+1)}-q^{(k+1)}$ 

9. $b_{z}^{(k+1)}=b_{z}^{(k)}+K z^{(k+1)}-u^{(k+1)}$
10. $k=k+1$
11. endwhile
12. return $z$

\section{Experimental results}

In this section, we present some numerical results to illustrate the performance of the proposed model for Poisson noise removal. In order to prove the efficiency of the proposed model, we compare our reconstruction results with those of the mentioned models: TV-model, HTV-model and FTVmodel. The compared models are implemented by the split Bregman method. We performed all experiments under MATLAB and Windows 10 on a PC with an Intel Core (TM) i5 CPU at $2.4 \mathrm{GHz}$ and 8 GB of RAM. Empirically, all images are processed with the equivalent parameters $\alpha=1.5, \mu_{1}=0.6$, $\mu_{2}=0.4, \rho_{1}=0.01, \rho_{2}=0.01, \rho_{3}=0.01, \theta=0.0001$. We set the stopping condition for Algorithm 1: $\varepsilon=0.00004$ and $N=500$. The observed images in our experiments are simulated as follows. Poisson noise is data dependent, the noise level of the observed images depends on the pixel intensity value. To test different noise levels, the noisy image is simulated by adding Poisson with some fixed value Peak. The test images are shown in Fig. 1.

The peak signal-to-noise ratio (PSNR) used in comparison are defined as follows:

$$
P S N R=10 \log _{10}\left(\frac{255^{2} \cdot M N}{\left\|u^{*}-u\right\|_{2}^{2}}\right),
$$

where $M$ and $N$ are the number of image pixels in rows and columns; $u, u^{*}$ are the original image, the reconstructed or noisy image accordingly. We also use other popular measure called structural similarity index measure (SSIM) which allows us to get more consistent with human visual characteristics [39]:

$$
\operatorname{SSIM}\left(u, u^{*}\right)=\frac{\left(2 \mu_{u} \mu_{u^{*}}+c_{1}\right)\left(2 \sigma_{u, u^{*}}+c_{2}\right)}{\left(\mu_{u}^{2}+\mu_{u^{*}}^{2}+c_{1}\right)\left(\sigma_{u}^{2}+\sigma_{u^{*}}^{2}+c_{2}\right)},
$$

where $\mu_{u}, \mu_{u^{*}}$ are the means of $u, u^{*}$ respectively; $\sigma_{u}$, $\sigma_{u^{*}}$ - their standard deviations; $\sigma_{u, u^{*}}$ - the covariance of two images $u$ and $u^{*} ; c_{1}, c_{2}$ are positive constants.

We first deal with Image denoising. In this case, $K$ is an identity matrix. In Figs. 2 and 3, we aim
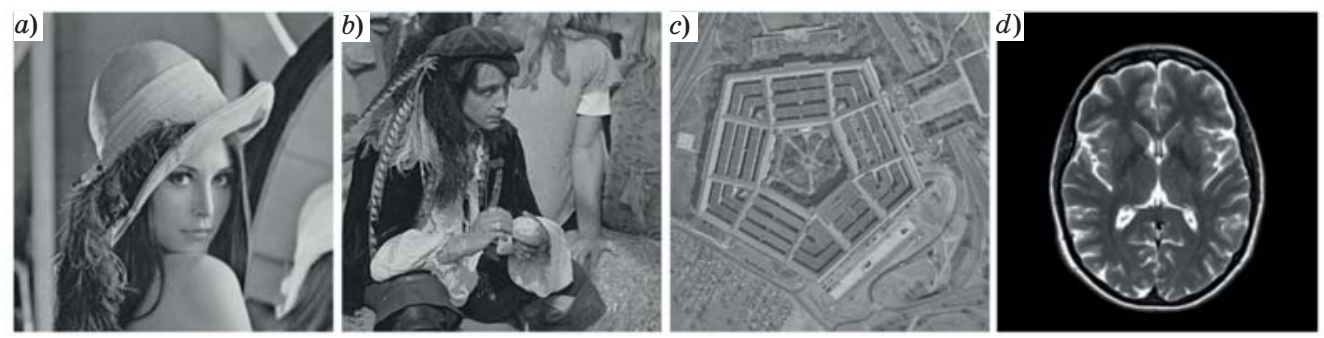

Fig. 1. Test images: $a-$ Lena; $b-$ Man; $c-$ Aerial; $d-$ MRI
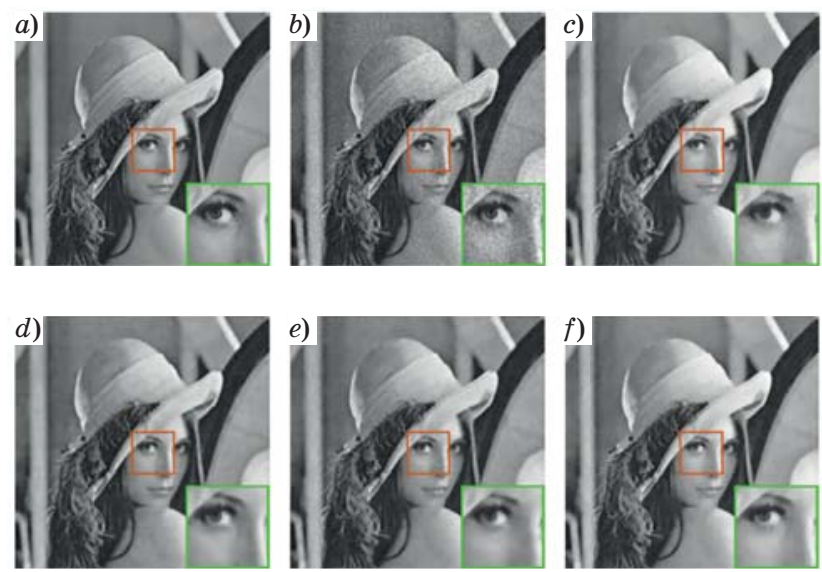

Fig. 2. Image "Lena". Recovered images of different methods for image denoising with Peak = 255: $a$ - original image; $b-$ noisy image; $c-\mathrm{TV} ; d-\mathrm{HTV} ; e-\mathrm{FTV}$; $f$ - ours
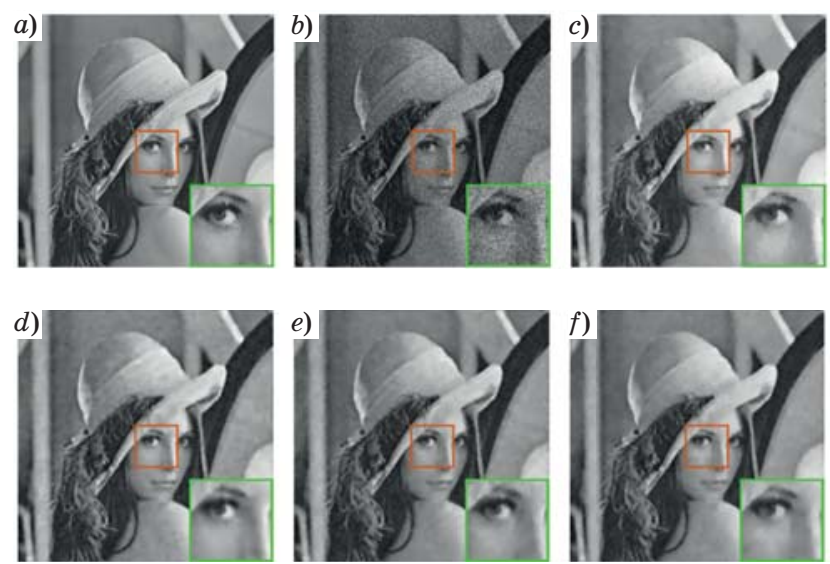

Fig. 3. Image "Lena". Recovered images of different methods for image denoising with Peak =100: $a$ - original image; $b-$ noisy image; $c-\mathrm{TV} ; d-\mathrm{HTV} ; e-\mathrm{FTV}$; $f$ - ours 
to restore the image "Lena" corrupted by Poisson noise with $P e a k=255$ and $P e a k=100$, respectively. In these figures, we also present the zoom-in on small details of the recovered images.

We see that TV-model is efficient for removing noise and simultaneously preserving the edges. However, the numerous artifact exists in result image recovered by TV-model. Meanwhile, the HTVmodel and FTV-model can lead to edge blurring when image denoising. The images recovered by our model are more smooth and distinct than those of another three approaches.

In Figs. 4, 6 and 8, we show the results of compared methods for noise levels $P e a k=100$. In Figs. 4, $b, 6, b$, and $8, b$, we represent the noisy images. In the others, Figs. 4, $c-f, 6, c-f, 8, c-f$, we show respectively the reconstructions given by TV, HTV, FTV and our proposed approach. In Figs. 5, 7 and 9, we show the zoomed details of the original images, observed images and the zoomed details of the re-
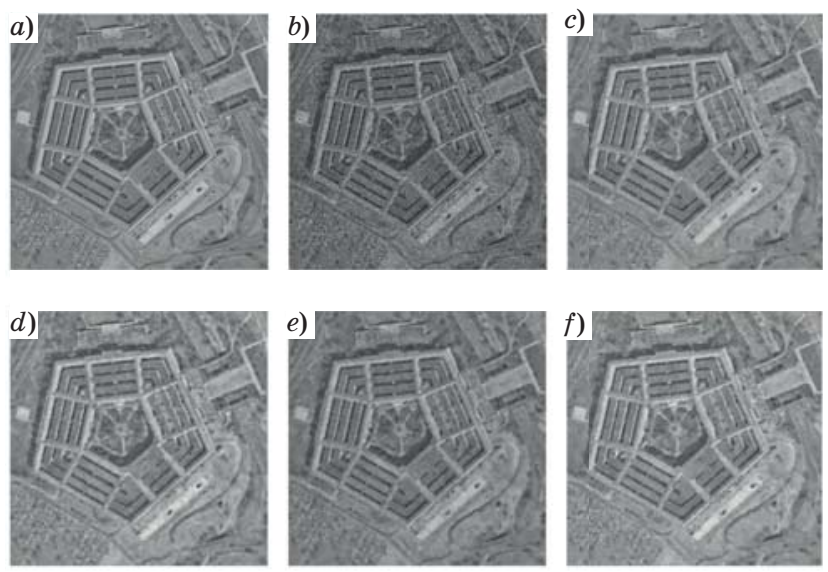

Fig. 4. Image "Aerial”. Recovered images of different methods for image denoising with $P e a k=100: a-$ original image; $b-$ noisy image; $c-\mathrm{TV} ; d-\mathrm{HTV} ; e-\mathrm{FTV}$; $f$ - ours
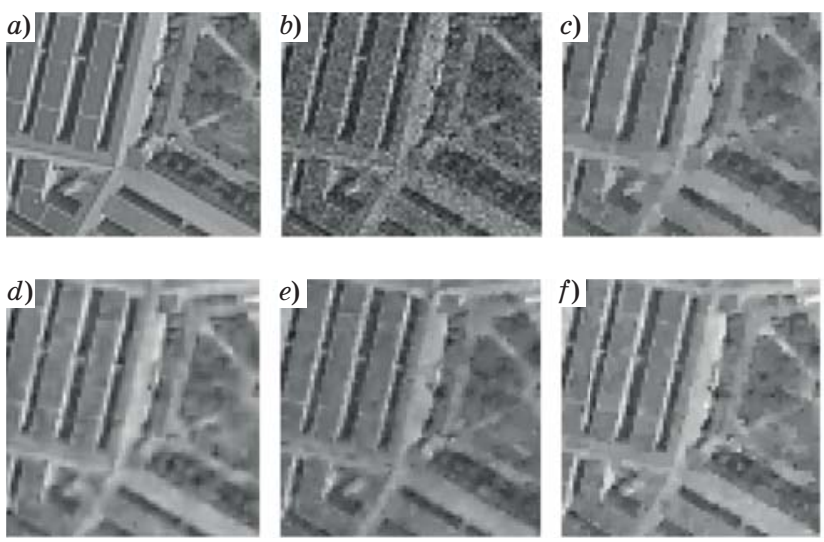

- Fig. 5. Image "Aerial". The zoomed-in details of the recovered images in Fig. 4: $a$ - original image; $b$ - noisy image; $c-\mathrm{TV} ; d-\mathrm{HTV} ; e-\mathrm{FTV} ; f-$ ours stored images respectively in Figs. 4, 6 and 8. From the details in Figs. 5, 7 and 9, we can see that the our model can get better visual improvement than the others. In Tables 1 and 2, we show the comparison results in terms of SSIM and PSNR (the best result is highlighted in bold). We can clearly see that our method outperforms the other relative methods for restoring images damaged by Poisson noise.

In the case of image deblurring and denoising, we consider blurred images degraded by Poisson noise. For simulation, we use the Gaussian blur with a window size $5 \times 5$ and standard deviation of 1 . After the blurring operation, we degrade the images by Poission noise with Peak =100. In Fig. 10, we perform simultaneously image deblurring and denoising on image "Lena". Fig. 10, $b$ denotes corrupted image. In Fig. 10, $c-f$, we show respectively the reconstructions given by TV, HTV, FTV and our approach.
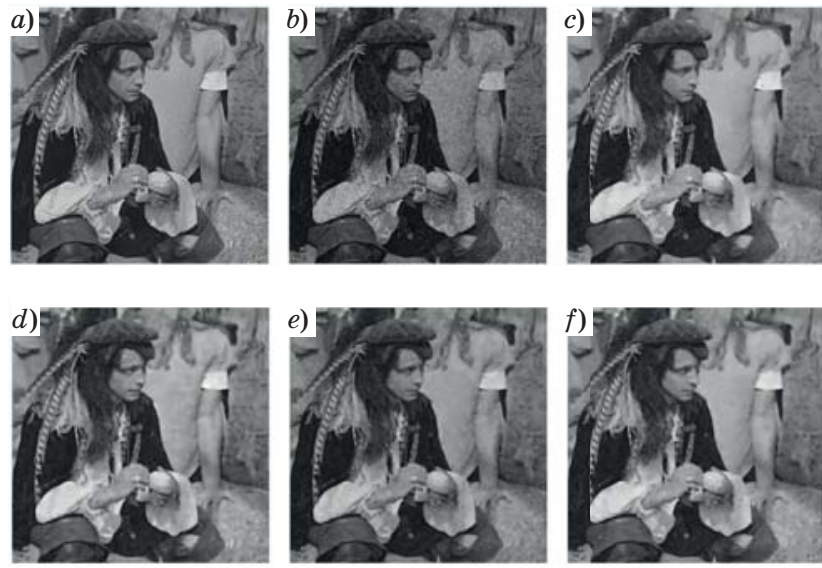

- Fig. 6. Image "Man". Recovered images of different methods for image denoising with $P e a k=100: a-$ original image; $b-$ noisy image; $c-\mathrm{TV} ; d-\mathrm{HTV} ; e-\mathrm{FTV}$; $f-$ ours
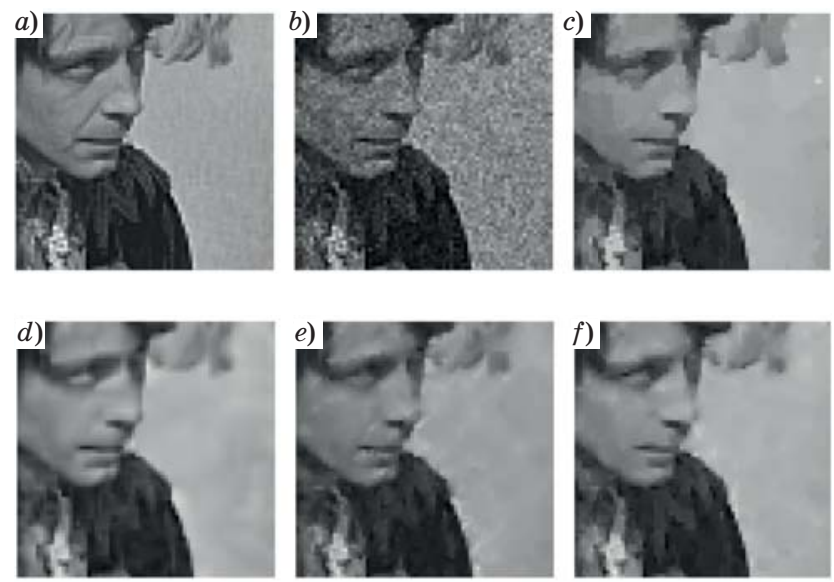

- Fig. 7. Image "Man". The zoomed-in details of the recovered images in Fig. 6: $a$ - original image; $b$ - noisy image; $c-\mathrm{TV} ; d-\mathrm{HTV} ; e-\mathrm{FTV} ; f-$ ours 

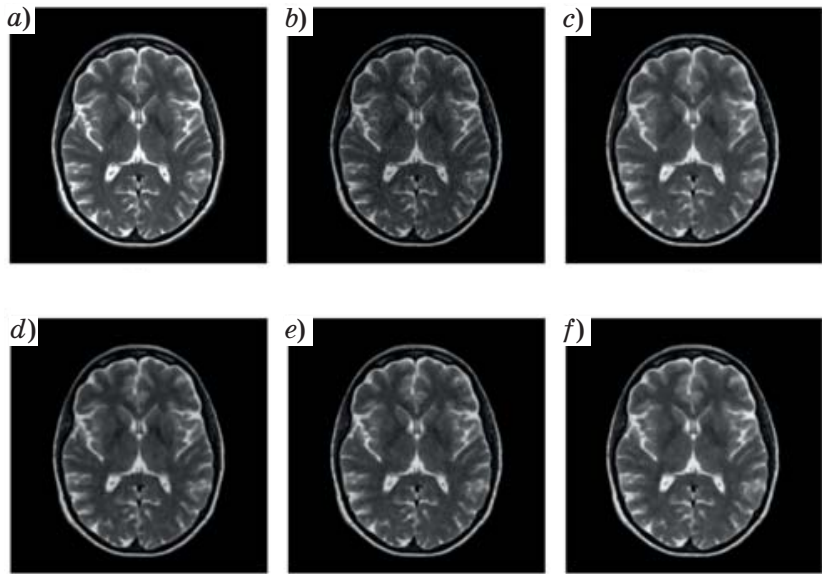

- Fig. 8. Image "MRI". Recovered images of different methods for image denoising with Peak =100: $a$ - original image; $b$ - noisy image; $c-\mathrm{TV} ; d-\mathrm{HTV} ; e-\mathrm{FTV} ; f$ - ours

- Table 1. PSNR values for recovered images given by the compared methods with various noisy levels

\begin{tabular}{|c|c|c|c|c|c|}
\hline \multirow{2}{*}{$\begin{array}{c}\text { Noise } \\
\text { level } \\
\text { Peak }\end{array}$} & Noisy & TV & HTV & FTV & Ours \\
\cline { 2 - 6 } Lena \\
\hline 255 & 26.9200 & 31.1786 & 30.9261 & 31.0504 & $\mathbf{3 1 . 8 6 4 0}$ \\
\hline 100 & 22.8909 & 29.1995 & 28.8624 & 29.2134 & $\mathbf{2 9 . 4 6 9 4}$ \\
\hline \multicolumn{6}{|c|}{ Man } \\
\hline 255 & 27.6436 & 29.6582 & 29.5528 & 29.8153 & 30.1140 \\
\hline 100 & 23.5718 & 26.5633 & 26.2971 & 26.7400 & $\mathbf{2 6 . 8 9 6 0}$ \\
\hline \multicolumn{7}{|c|}{ Aerial } \\
\hline 255 & 26.7701 & 28.4530 & 28.2417 & 28.4966 & $\mathbf{2 9 . 0 0 6}$ \\
\hline 100 & 22.6521 & 25.8654 & 25.7517 & 25.6930 & $\mathbf{2 6 . 2 9 6 6}$ \\
\hline \multicolumn{7}{|c|}{ MRI } \\
\hline 255 & 30.1130 & 30.7451 & 30.7111 & 30.8487 & 30.9878 \\
\hline 100 & 26.9175 & 28.9371 & 28.9151 & 28.9780 & $\mathbf{2 9 . 2 7 7 2}$ \\
\hline
\end{tabular}
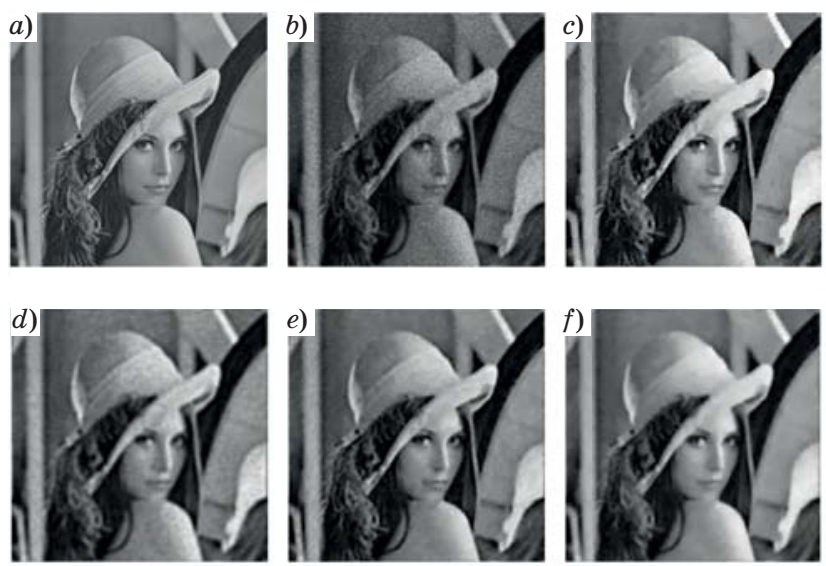

- Fig. 10. Image "Lena". Recovered results for the test images: $a$ - original image; $b-$ noisy image; $c-\mathrm{TV}$; $d$-HTV; $e-$ FTV; $f$ - ours
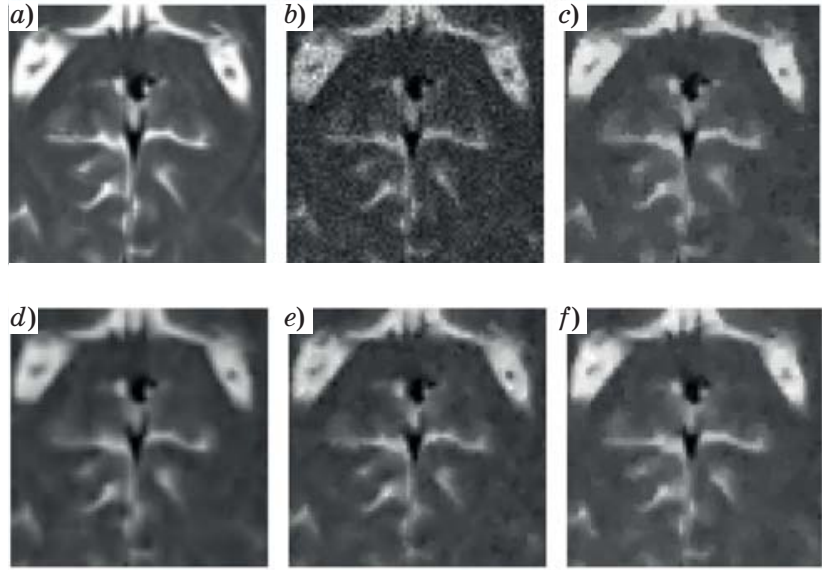

- Fig. 9. Image "MRI". The zoomed-in details of the recovered images in Fig. (8): $a$ - original image; $b$ - noisy image; $c-\mathrm{TV} ; d-\mathrm{HTV} ; e-\mathrm{FTV} ; f-$ ours

- Table 2. SSIM values for recovered images given by the compared methods with various noisy levels

\begin{tabular}{|c|c|c|c|c|c|}
\hline \multirow{2}{*}{$\begin{array}{l}\text { Noise } \\
\text { level } \\
\text { Peak }\end{array}$} & \multicolumn{5}{|c|}{ SSIM } \\
\hline & Noisy & $\mathrm{TV}$ & HTV & FTV & Ours \\
\hline \multicolumn{6}{|c|}{ Lena } \\
\hline 255 & 0.6721 & 0.8794 & 0.8812 & 0.8780 & 0.8891 \\
\hline 100 & 0.5234 & 0.8368 & 0.8423 & 0.8411 & 0.8505 \\
\hline \multicolumn{6}{|c|}{ Man } \\
\hline 255 & 0.8028 & 0.8770 & 0.8731 & 0.8713 & 0.8835 \\
\hline 100 & 0.6578 & 0.7830 & 0.7784 & 0.7862 & 0.7986 \\
\hline \multicolumn{6}{|c|}{ Aerial } \\
\hline 255 & 0.8277 & 0.8649 & 0.8585 & 0.8645 & 0.8805 \\
\hline 100 & 0.7154 & 0.7787 & 0.7639 & 0.7647 & 0.7882 \\
\hline \multicolumn{6}{|c|}{ MRI } \\
\hline 255 & 0.9025 & 0.9401 & 0.9427 & 0.9344 & 0.9484 \\
\hline 100 & 0.8416 & 0.9107 & 0.9114 & 0.9095 & 0.9272 \\
\hline
\end{tabular}
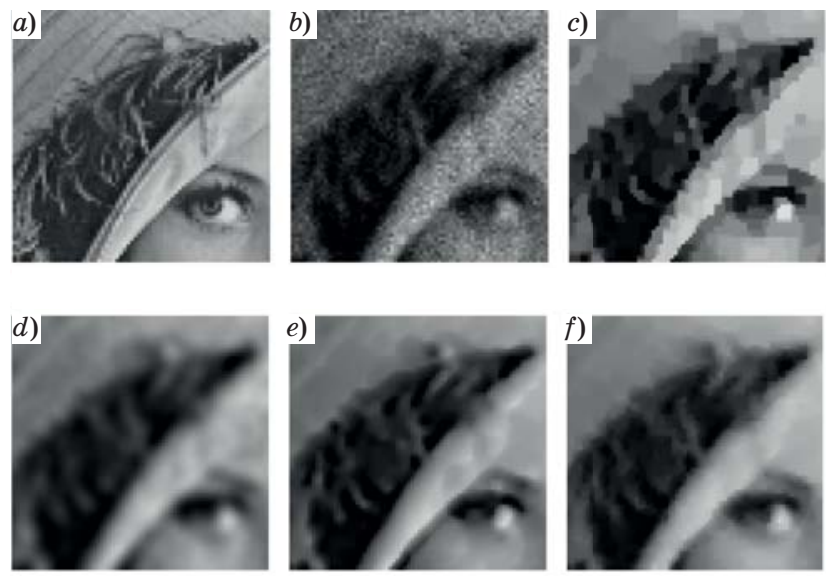

- Fig. 11. Image "Lena". The zoomed-in details of the recovered images in Fig. 10: $a$ - original image; $b-$ noisy image; $c-\mathrm{TV} ; d-\mathrm{HTV} ; e-\mathrm{FTV} ; f-$ ours 
- Table 3. PSNR values for recovering blurred images with noisy level Peak $=100$

\begin{tabular}{|c|c|c|c|c|c|}
\hline \multirow{2}{*}{ Image } & \multicolumn{5}{|c|}{ PSNR } \\
\cline { 2 - 6 } & Noisy & TV & HTV & FTV & Ours \\
\hline Lena & 20.6275 & 22.7049 & 22.5994 & 22.8684 & $\mathbf{2 3 . 3 1 3 3}$ \\
\hline Man & 19.4880 & 20.5501 & 20.0390 & 20.4229 & $\mathbf{2 0 . 6 9 1 6}$ \\
\hline Aerial & 18.3362 & 18.7176 & 18.9762 & 18.8378 & $\mathbf{1 9 . 4 5 3 7}$ \\
\hline MRI & 24.3167 & 24.9252 & 24.231 & 24.6373 & $\mathbf{2 5 . 0 1 6 6}$ \\
\hline
\end{tabular}

Table 4. SSIM values for recovering blurred images with noisy level Peak $=100$

\begin{tabular}{|c|c|c|c|c|c|}
\hline \multirow{2}{*}{ Image } & \multicolumn{5}{|c|}{ SSIM } \\
\cline { 2 - 6 } & Noisy & TV & HTV & FTV & Ours \\
\hline Lena & 0.4354 & 0.7503 & 0.7190 & 0.7647 & $\mathbf{0 . 7 7 3 9}$ \\
\hline Man & 0.4748 & 0.5702 & 0.5432 & 0.5853 & $\mathbf{0 . 5 9 9 4}$ \\
\hline Aerial & 0.5220 & 0.6047 & 0.5430 & 0.6074 & $\mathbf{0 . 6 2 1 4}$ \\
\hline MRI & 0.7956 & 0.8642 & 0.8527 & 0.8690 & $\mathbf{0 . 8 7 6 0}$ \\
\hline
\end{tabular}

Meanwhile, Fig. 11 shows enlarged images recovered by four methods in Fig. 10. In Tables 3 and 4 , we report the quantitative measures of PSNR and SSIM values for different images and compared methods. The Figures and Tables demonstrate again the effectiveness of our proposed method for image reconstruction under Poisson noise even in presence of blur.

\section{Conclusions}

In this paper, we have researched the hybrid regularizers model, combining the fractional-order and first-order TV for denoising images corrupted by Poisson noise. Computationally, an extended split Bregman method is employed for solving the proposed optimization problem. Finally, compared with the existing state-of-the-art models, the ex- periments demonstrate the efficiency of the proposed method.

\section{Acknowledgments}

Pham Cong Thang (corresponding author) would like to thank his colleagues at IT Faculty, DUT, for their helpful comments. The authors also thank reviewers and Editors for their insightful comments and suggestions.

\section{Financial support}

This work is supported by The Murata Science Foundation and The University of Danang, University of Science and Technology, code number of Project T2020-02-07MSF.

\section{References}

1. Hasinoff S. W. Photon, poisson noise. In: Computer vision. Ed. K. Ikeuchi. Boston, MA, Springer US, 2014. Pp. 608-610. doi:10.1007/978-0-387-31439$6 \_482$

2. Zhang Y., Zhu Y., Nichols E., Wang Q., Zhang S., Smith C., Howard S. A Poisson - Gaussian denoising dataset with real fluorescence microscopy images. Proceedings of the IEEE/CVF Conference on Computer Vision and Pattern Recognition (CVPR), 2019, pp. 11710-11718.

3. Meiniel W., Olivo-Marin J., Angelini E. D. Denoising of microscopy images: A review of the state-of-theart, and a new sparsity-based method. IEEE Transactions on Image Processing, 2018, vol. 27, no. 8, pp. 3842-3856. doi:10.1109/TIP.2018.2819821

4. Bubba T. A., Porta F., Zanghirati G., Bonettini S. A nonsmooth regularization approach based on shearlets for Poisson noise removal in ROI tomography. Applied Mathematics and Computation, 2018, vol. 318, no. 1, pp. 131-152. doi:10.1016/j.amc.2017.09.001

5. Zeng G. L, Lv L., Huang Q. Poisson-noise weighted filter for time-of-flight positron emission tomography. Visual Computing for Industry, Biomedicine and
Art 2020 , vol. 3, Article number: 10, 4 p. doi:10.1186/ s42492-020-00048-8

6. Ofek E. O., Zackay B. Optimal matched filter in the low-number count Poisson noise regime and implications for X-ray source detection. The Astronomical Journal, 2018, vol. 155, no. 4, 8 p. doi:10.3847/1538$3881 / a a b 265$

7. Yagoub B., Ibrahem H., Salem A., Suh J. W., Kang H. S. X-ray image denoising for Cargo Dual Energy Inspection System. International Conference on Electronics, Information, and Communication (ICEIC), 2021, pp. 1-4. doi:10.1109/ICEIC51217.2021.9369786

8. Pham C. T., Gamard G., Kopylov A., Tran T. T. T. An algorithm for image restoration with mixed noise using total variation regularization. Turkish Journal of Electrical Engineering and Computer Sciences, 2018, vol. 26, no. 6, pp. 2831- 2845. doi:10.3906/elk-1803-100

9. Kockentiedt S., Tonnies K. D., Gierke E., Nico D., Thim C., Plitzko S. Poisson shot noise parameter estimation from a single scanning electron microscopy image. Proc. SPIE 8655, Image Processing: Algorithms and Systems XI, 2013, 86550N. doi:10.1117/ 12.2008374

10. Seki T., Ikuhara Y., Shibata N. Theoretical framework of statistical noise in scanning transmission 
electron microscopy. Ultramicroscopy, 2018, vol. 193, pp. 118-125. doi:10.1016/j.ultramic.2018.06.014

11. Sanders T. The potential for Poisson image reconstruction models for electron tomography. Proceedings of SPIE Volume 10772, Unconventional and Indirect Imaging, Image Reconstruction, and Wavefront Sensing, 2018, 1077200. doi:10.1117/12.2321700

12. Diwakar M., Kumar M. A review on CT image noise and its denoising. Biomedical Signal Processing and Control, 2018, vol. 42, pp. 73-88. doi:10.1016/j.bspc. 2018.01.010

13. Pham C. T., Tran T. T. T., Phan T. D. K., Dinh V. S., Pham M. T., Nguyen M. H. An adaptive algorithm for restoring image corrupted by mixed noise. Cybernetics and Physics, 2019, vol. 8, no. 2, pp. 73-82. doi:10.35470/2226-4116-2019-8-2-73-82

14. Kirti T., Jitendra K., Ashok S. Poisson noise reduction from X-ray images by region classification and response median filtering. Sadhana, 2017, vol. 42, no. 6, pp. 855-863. doi:10.1007/s12046-017-0654-4

15. Sun Y., Liu X., Cong P., Li L., Zhao Z. Digital radiography image denoising using a generative adversarial network. Journal of X-Ray Science and Technology, 2018, vol. 26, pp. 523-534. doi:10.3233/XST-17356 IOS Press

16. Bal A., Banerjee M., Chaki R., Sharma P. An efficient method for PET image denoising by combining multi-scale transform and non-local means. Multimedia Tools and Applications, 2020, vol. 79, pp. 2908729120. doi:10.1007/s11042-020-08936-0

17. Chen H., Fu S., Wang H., Zhai L., Wang F., Li Y. Fuzzy singular value shrinkage for Poisson image denoising. Journal of Modern Optics, 2019, vol. 66, pp. 1569-1578. doi:10.1080/09500340.2019.1648895

18. Kayyar S. H., Jidesh P. Non-local total variation regularization approach for image restoration under a Poisson degradation. Journal of Modern Optics, 2018, vol. 65 , no. 19, pp. 2231-2242. doi:10.1080/095 00340.2018.1506058

19. Zhang M., Zhang F., Liu Q., Wang S. VST-Net: Variance-stabilizing transformation inspired network for Poisson denoising. Journal of Visual Communication and Image Representation, 2019, vol. 62, pp. 12-22. doi:10.1016/j.jvcir.2019.04.011

20. Bindilatti A. A., Vieira M. A. C., Mascarenhasac N. D. A. Poisson Wiener filtering with non-local weighted parameter estimation using stochastic distances. Signal Processing, 2018, vol. 144, pp. 68-76. doi:10.1016/j.sigpro.2017.10.001

21. Niknejad M., Figueiredo M. A. T. Poisson image denoising using best linear prediction: a post-processing framework. European Signal Processing Conference, 2018, pp. 2230-2234. doi:10.23919/EUSIPCO.2018.8553220

22. Bodduna K., and Weickert J. Poisson noise removal using multi-frame 3D block matching. 8th European Workshop on Visual Information Processing (EUVIP), 2019, pp. 58-63. doi:10.1109/EUVIP47703.2019.8946209
23. Pham C. T, Tran T. T. T. An algorithm for hybrid regularizers based image restoration with Poisson noise. Kybernetika, 2021, vol. 57, no. 3, pp. 446-47. doi: 10.14736/kyb-2021-3-0446

24.Le T., Chartrand R., Asaki T. A variational approach to constructing images corrupted by Poisson noise. Journal of Mathematical Imaging and Vision, 2007, vol. 27, pp. 257-263. doi:10.1007/ s10851-007-0652-y

25. Liu X., Huang L. Total bounded variation-based Poissonian images recovery by split Bregman iteration. Mathematical Methods in the Applied Sciences, 2012, vol. 35, no. 5, pp. 520-529. doi:10.1002/mma.1588

26.Zhang J., Ma M., Wu Z., Deng C. 2019. High-order total bounded variation model and its fast algorithm for Poissonian image restoration. Mathematical Problems in Engineering, 2019, vol. 2019, 11 p. doi:10.1155/2019/2502731

27. Zhang J., Wei Z., Xiao L. Adaptive fractional-order multi-scale method for image denoising. Journal of Mathematical Imaging and Vision, 2012, vol. 43, pp. 39-49. doi:10.1007/s10851-011-0285-z

28. Chen D., Chen Y. Q., Xue D. Fractional-order total variation image denoising based on proximity algorithm. Applied Mathematics and Computation, 2015, vol. 257, pp. 537-545. doi:10.1016/j.amc.2015.01.012

29. Chowdhury M. R., Zhang J., Qin J., Lou Y. Poisson image denoising based on fractional-order total variation. Inverse Problems and Imaging, 2020, vol. 14, no. 1, pp. 77-96. doi:10.3934/ipi.2019064

30. Zhao W., Lu H. Medical image fusion and denoising with alternating sequential filter and adaptive fractional order total variation. IEEE Transactions on Instrumentation and Measurement, 2017, vol. 66, no. 9, pp. 2283-2294. doi:10.1109/TIM.2017.2700198

31. Huang G., Chen Q., Men T., Zhang X. Q., Qin H. Y., $\mathrm{Xu}$ L. Application in image denoising using fractional total variation theory. The Proceedings of the International Conference on Sensing and Imaging, 2017, pp. 211-221. doi:10.1007/978-3-319-91659-0_15

32. Pham C. T., Tran T. T. T, Gamard G. An efficient total variation minimization method for image restoration. Informatica, 2020, vol. 31, no. 3, pp. 539-560. doi:10.15388/20-INFOR407

33. Tran T. T. T., Pham, C. T., Kopylov A. V., Nguyen V. N. An adaptive variational model for medical images restoration. ISPRS International Archives of the Photogrammetry, Remote Sensing and Spatial Information Sciences, 2019, vol. XLII-2/W12, pp. 219-224. doi:10.5194/isprs-archives-XLII-2-W12-219-2019

34. Pham C. T., Tran T. T. T., Nguyen T. C., Vo D. H. Second-order total generalized variation based model for restoring images with mixed Poisson - Gaussian noise. Informatsionno-upravliaiushchie sistemy [Information and Control Systems], 2021, no. 2, pp. 2032. doi:10.31799/1684-8853-2021-2-20-32

35. Pham C. T, Tran T. T. T., Vo D. H., Vo D.-H. A hybrid regularizers model for multiplicative noise removal. 
Cybernetics and Physics, 2021, vol. 10, no. 1, pp. 4050. doi:10.35470/2226-4116-2021-10-1-40-50

36. Goldstein T., Osher S. The split Bregman method for $L_{1}$-regularized problems. SIAM Journal on Imaging Sciences, 2009, vol. 2, no. 2, pp. 323-343. doi:10. $1137 / 080725891$

37. Zhang C. C., Wang Y., Xiao H. B. Applying improved split Bregman method to fluorescence microscopic image restoration. Computer Science, Technology and Application, 2016, pp. 54-60. doi:10.1142/ 9789813200449_0008
38. Yang Y., Tian D., Jia W., Shu X., Wu B. Split Bregman method based level set formulations for segmentation and correction with application to MR images and color images. Magnetic Resonance Imaging, 2019, vol.57, pp.50-67.doi:10.1016/j.mri.2018.10.005

39. Bovik A. C., Wang Z. Modern Image Quality Assessment, Synthesis Lectures on Image, Video, and Multimedia Processing. Morgan and Claypool Publishers, 2006. 156 p. doi:10.2200/S00010ED1V01Y200508IVM003

УДК 004.93

doi:10.31799/1684-8853-2021-5-10-19

Комбинированная полная вариация первого и дробного порядка для удаления пуассоновского шума на цифровых изображениях

Фам Конг Тханга ${ }^{\mathrm{P} h D}$, преподаватель, orcid.org/0000-0002-6428-102X, pcthang@dut.udn.vn

Чан Тхи Тху Тхао ${ }^{\sigma}$ магистр, преподаватель, orcid.org/0000-0001-7705-2405

Фам Минь Туан ${ }^{\mathrm{a}}, \mathrm{PhD}$, преподаватель, orcid.org/0000-0001-9843-9676

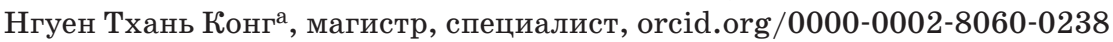

ауниверситет Дананга - Университет науки и техники, Нгуен Лыонг Банг, 54, Дананг, 550000, Вьетнам

бУниверситет Дананга - Университет экономики, Нгу Хань Шон, 71, Дананг, 550000, Вьетнам

Введение: известно множество методов для решения проблемы восстановления изображений с помощью пуассоновского шума. Одним из популярных подходов к реконструкции пуассоновского изображения является метод, основанный на полной вариации. С помощью этого метода можно получить весьма резкие границы и визуально четкие изображения, но он приводит к образованию кусочно-постоянных областей в результирующих изображениях. Цель: разработка адаптивной модели на основе полных вариаций для реконструкции изображений, искаженных пуассоновским шумом, и алгоритма решения задачи оптимизации. Результаты: предложен эффективный метод для восстановления изображений, искаженных пуассоновским шумом. На базе байесовской структуры предложена адаптивная модель, основанная на комбинации полной вариации первого порядка и полной вариации дробного порядка. Восстановление изображения на основе модели полной вариации первого порядка эффективно для шумоподавления и одновременно сохранения острых границ. Однако метод полной вариации первого порядка обычно вызывает проблемы с артефактами в полученных результатах. Чтобы избежать этого недостатка, использованы модели полной вариации высокого порядка, одна из которых является основанной на полной вариации дробного порядка для восстановления изображений. В модели полной вариации дробного порядка производные имеют порядок больше или равный единице. Это приводит к удобству вычислений с компактной дискретной формой. Но методы, основанные на полной вариации дробного порядка, могут вызвать размытие изображения. Таким образом, предложенная модель включает в себя преимущества двух моделей регуляризации полной вариации и существенно влияет на восстановление изображения с сохранением границ. Для решения рассматриваемой оптимизационной задачи используется метод Split Bregman. Приведены экспериментальные результаты, демонстрирующие эффективность предложенного метода. Практическая значимость: предлагаемый метод позволяет восстанавливать пуассоновские изображения с сохранением границ. Представленное численное моделирование наглядно демонстрирует конкурентоспособность предложенной модели для восстановления изображений, деградированных пуассоновским шумом. Обсуждение: из результатов экспериментов видно, что предложенный алгоритм эффективен в шумоподавлении и сохранении границ изображения. Однако взвешенные параметры, существующие в предложенной модели, не выбирались автоматически на каждой итерации предложенного алгоритма, что требует дополнительных исследований.

Ключевые слова - полная вариация, восстановление изображения, пуассоновский шум, метод минимизации.

Для цитирования: Pham С. Т., Tran Т. Т. Т., Pham М. Т., Nguyen T. С. Combined total variation of first and fractional orders for Poisson noise removal in digital images. Информационно-управляющие системы, 2021, № 5, с. 10-19. doi:10.31799/1684-8853-20215-10-19

For citation: Pham C. T., Tran T. T. T., Pham M. T., Nguyen T. C. Combined total variation of first and fractional orders for Poisson noise removal in digital images. Informatsionno-upravliaiushchie sistemy [Information and Control Systems], 2021, no. 5, pp. 10-19. doi:10.31799/1684-8853-2021-5-10-19 